\title{
Implementation and assessment of a pediatric point-of-care ultrasound training course in Japan: a pilot study
}

\author{
Takaaki Mori $^{1} \mathbb{D} \cdot$ Osamu Nomura ${ }^{2} \cdot$ Hirokazu Takei $^{3} \cdot$ Shinichi Fukuhara ${ }^{4} \cdot$ Ko Ichihashi $^{5}$
}

Received: 15 June 2021 / Accepted: 17 August 2021 / Published online: 22 October 2021

(c) The Author(s), under exclusive licence to The Japan Society of Ultrasonics in Medicine 2021

\begin{abstract}
Purpose The popularity of point-of-care ultrasound (POCUS) has led to the creation of educational guidelines for its use. In Japan, however, a comprehensive training course for POCUS use in pediatric emergency medicine has yet to be developed. The present study aimed to implement a pilot course for pediatric POCUS training in Japan and to compare participants' self-efficacy level before and after the course.

Methods A half-day training course in pediatric POCUS was implemented at a meeting of the Japan Society of Point-ofCare Ultrasound. A standardized training course, including pre-learning materials, live lectures, and hands-on sessions, was developed based on the US consensus educational guidelines. Physicians interested in pediatric POCUS were recruited for participation and completed a self-evaluation survey before and after the course to access their background, self-efficacy in performing selected ultrasound procedures before and after the course, and their overall satisfaction with the course.

Results In total, 31 physicians participated. Of these, 25 completed the survey. Ten participants were in post-graduate year (PGY) 1-2, 13 were in PGY 3-5, and eight were in PGY 6 or higher. The post-training self-efficacy score was significantly higher than the pre-course assessment score (86.0 [standard deviation (SD): 19.2] vs. 35.6 [SD 17.6], $p=<0.05$, mean difference: 49.6 [95\% confidence interval 39.6-61.2]). Furthermore, overall satisfaction with the course was high at 8.6 (SD 1.8). Conclusion The present study implemented a pilot training course in pediatric POCUS and found the participants' selfefficacy level to be significantly higher after the course.
\end{abstract}

Keywords Ultrasonography $\cdot$ Education $\cdot$ Child

Takaaki Mori

takaakimori001019@gmail.com

1 Division of Pediatric Emergency Medicine, Department of Pediatric Emergency and Critical Care Medicine, Tokyo Metropolitan Children's Medical Center, 2-8-29 Musashidai, Fuchu, Tokyo 183-8561, Japan

2 Department of Emergency and Disaster Medicine, Hirosaki University, 5 Zaifu-cho, Hirosaki, Aomori 035-8562, Japan

3 Department of Emergency Medicine, Hyogo Prefectural Kobe Children's Hospital, 1-6-7 Minatojimaminamicho, Chuo-ku, Kobe 650-0047, Japan

4 Department of Pediatrics, Hyogo Prefectural Awaji Medical Center, 1-1-137 Shioya, Sumoto, Hyogo 656-0021, Japan

5 Department of Pediatrics, Jichi Medical University Saitama Medical Center, 1-847 Amanuma-cho, Ohmiya-ku, Saitama 330-8503, Japan

\section{Introduction}

Point-of-care ultrasound (POCUS), a form of diagnostic and therapeutic ultrasonography (US) performed by physicians treating patients, is a popular choice in pediatric emergency medicine (PEM) [1] because of its ability to provide real-time imaging that enables physicians to make clinical decisions under time-sensitive conditions and because, unlike radiography or computed tomography, it does not involve radiation exposure, which can trigger secondary malignancies [2]. Thanks to its increasing popularity, in 2015 the American College of Emergency Physicians and the American Academy of Pediatrics published a consensus statement on its use [3], and in 2016 a systematic review of POCUS use in pediatric care proposed reporting guidelines for POCUS examinations [4]. Given its popularity, training courses in pediatric POCUS are being implemented worldwide, further corroborating the efficacy of this technique [5-13]. However, many of the 
training courses target specialists such as pediatric intensivists and pediatric cardiologists [7-10,12], and focus on cardiac and soft tissue US or US-guided nerve block $[6,9,11]$. Only one study focused on the effectiveness of comprehensive training in the use of pediatric POCUS and demonstrated an increase in the knowledge and confidence level of its users [13].

In contrast, in Japan, a large proportion of pediatricians and emergency physicians have access to US machines and can perform US examinations. Despite the preference commonly shown by pediatricians for POCUS, as witnessed by the frequent and informal courses held on its use [14], no descriptive studies of POCUS use among pediatricians in Japan have been done, nor has a standardized, comprehensive training course for pediatric POCUS use been developed, and data on its efficacy have yet to be documented. To address this omission, we implemented a comprehensive pilot training program for pediatric POCUS use by adapting the aforementioned consensus guidelines to the current clinical situation in Japan [3, 4, 15] and evaluated its effectiveness. The level of participants' satisfaction with the course was initially assessed in a pilot study in 2015, which demonstrated a high satisfaction rate [unpublished work]. The present study aimed to continue the inquiry by analyzing participants' assessment of self-efficacy in the course.

\section{Materials and methods}

The present before-and-after study investigated the selfefficacy level of participants in a pediatric POCUS training program conducted between August 2019 and January 2020 .

\section{Pediatric POCUS training course}

Instructors were recruited from different parts of Japan based on their previous experience in teaching US use at their respective institutions. They had at least 3 years of experience in using pediatric POCUS and previously completed the pediatric emergency US course certified by the World Interactive Network Focused on Critical Ultrasound (WINFOCUS) [16] or were certified by the Japan Society of Ultrasonics in Medicine. They then established a curriculum for pediatric POCUS training by discussion. Examples of POCUS applications in our courses were chosen by adapting previously published educational guidelines $[3,4,15]$ to the current clinical situation in Japan, as shown in Table 1. Because all the teaching objectives could not be covered in one course, it was decided that they should be distributed over several courses. Previous data from a questionnaire administered at a hands-on session during the 29th annual meeting of the Japanese Society of Emergency Pediatrics in 2015 [unpublished work] showed that abdominal and cardiac US were relatively common US applications for pediatricians in Japan, whereas ocular or musculoskeletal US was less frequently used. Both relatively common US applications such as cardiac and abdominal US, and less frequent but clinically important ones including musculoskeletal and ocular US, or US in shock management, were selected for

Table 1 Educational domains

\begin{tabular}{lc}
\hline Domains & Main learning objectives \\
\hline Pediatric FOCUS & $\begin{array}{c}\text { Acquisition of PLAX, PSAX, A4C, S4C, IVC, SCLAo views interpretation of LV systolic func- } \\
\text { tion, pericardial effusion, IVC, and simple assessment of congenital abnormalities }\end{array}$ \\
Pediatric abdominal ultrasound & $\begin{array}{c}\text { Identification of normal abdominal structures (i.e., liver, spleen, kidney) and typical abnormal } \\
\text { images (i.e., intussusception, acute appendicitis, hydronephrosis), performance of FAST (focused } \\
\text { assessment with sonography for trauma) } \\
\text { Recognition of normal airway/lung structures (i.e., trachea, esophagus, A-lines, B-lines) identifica- } \\
\text { tion of typical abnormal images (i.e., pneumonia, pneumothorax, interstitial syndrome) }\end{array}$ \\
Pediatric airway/lung ultrasound & $\begin{array}{c}\text { Recognition of normal musculoskeletal structures (i.e., skin, bone, nerve) identification of typical } \\
\text { abnormal images (i.e., fracture, cellulitis, abscess, subcutaneous foreign body) }\end{array}$ \\
Pediatric ocular ultrasound & $\begin{array}{c}\text { Recognition of normal ocular structures (i.e., iris, lens, optic nerve sheath) identification of typical } \\
\text { abnormal images (i.e., retinal detachment, papilledema) }\end{array}$ \\
Ultrasound-guided procedures in children & $\begin{array}{c}\text { Performance of ultrasound-guided procedures (i.e., vascular access, nerve block, foreign body } \\
\text { removal) }\end{array}$ \\
Ultrasound assessment for shock in children & \begin{tabular}{c} 
Differentiation of shock combining cardiac and lung ultrasound \\
\hline
\end{tabular}
\end{tabular}

FOCUS focused cardiac ultrasound, PLAX parasternal long axis view, PSAX parasternal short axis view, $A 4 C$ apical 4-chamber view, $S 4 C$ subcostal 4-chamber view, IVC inferior vena cava, $S C L A o$ supraclavicular long axis aorta view, $L V$ left ventricle 


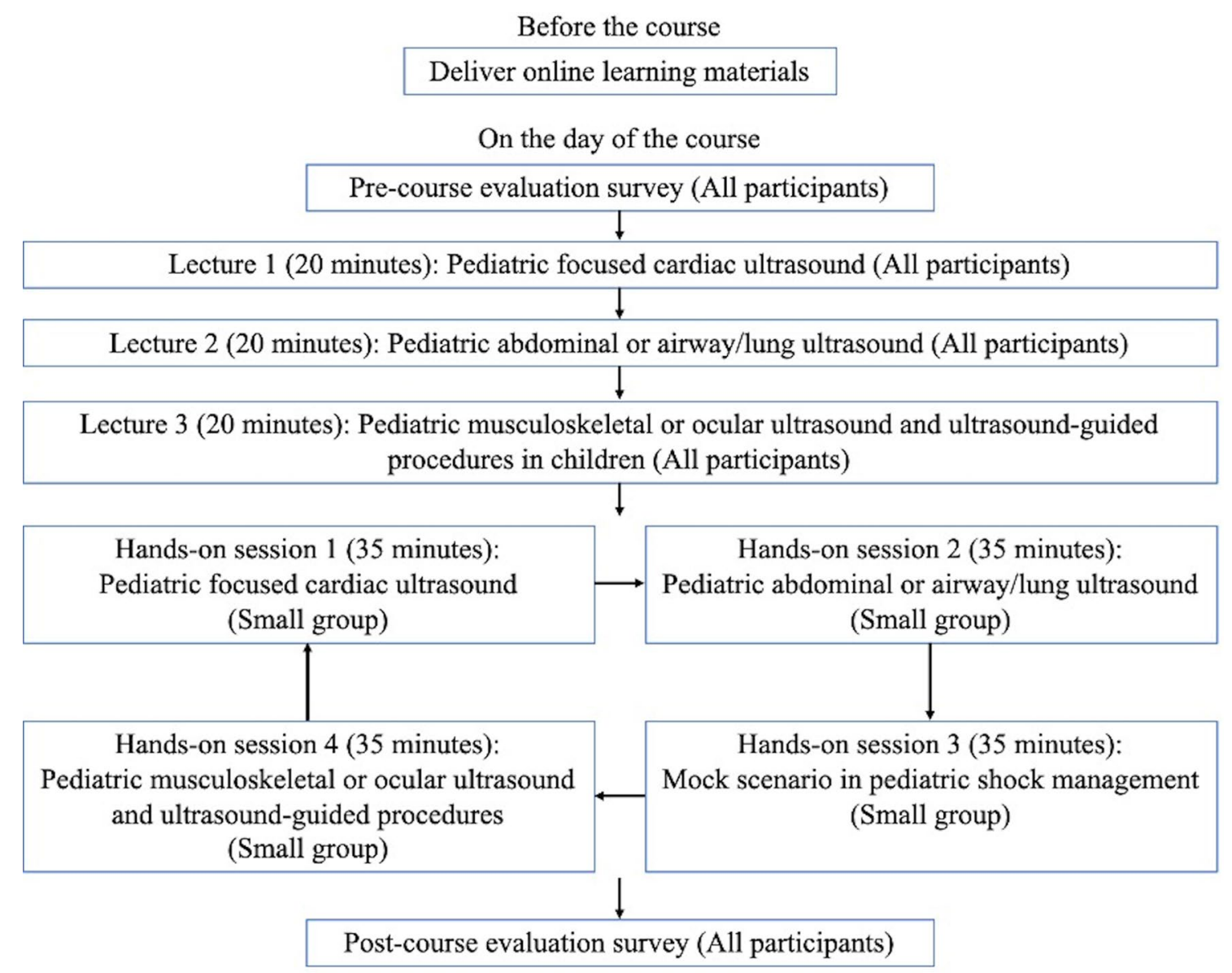

Fig. 1 Course flow. The present comprehensive pediatric POCUS training course was held at the 7th and 8th conferences of the Japan Society of Point-of-Care Ultrasound in August 2019 and January 2020, respectively

the course. The course was implemented at the 7th and 8th conferences of the Japan Society of Point-of-Care Ultrasound in August 2019 and January 2020, respectively, and was held separately from the main conference. The training consisted of a half-day tutorial, including three lectures (20 min each) and four hands-on training sessions (35 min each) with a trainer-to-learner ratio of 1:4-5 (Fig. 1). Of the three lectures, one dealt with cardiac US, and of the four hands-on sessions, two dealt with cardiac US and shock management. The remaining two lectures and hands-on sessions were based on the aforementioned consensus guidelines and dealt with POCUS use in abdominal, airway/lung, musculoskeletal, and ocular examinations, and US-guided procedures, as described in Table 2 and Fig. 1.

LOGIQ $^{\mathrm{TM}}$ e (GE Healthcare, Japan), Xario200G (Cannon Medical Systems Corporation, Japan), Noblus (Hitachi Medical Systems, Japan), and Sonosite X-Porte (FUJIFILM, SonoSite Inc., Japan) were used for scanning. For cardiac, airway/lung, and ocular US, young adult volunteers were used for scanning, and for abdominal and musculoskeletal US, a pediatric anatomical simulator (Pediatric FAST/Acute
Abdomen Phantom; Kyoto Kagaku Co., Ltd.) and a handmade model using a chicken leg were used together with volunteers. The conference organizer explained the contents of the training course to the volunteers and obtained their oral consent prior to participation. For US-guided procedures such as US-guided peripheral line placement and USguided nerve block, simulators (peripherally inserted central venous catheter simulator [PICC simulator], Kyoto Kagaku Co., Ltd.; CAE blue phantom, Gadelius Medical K.K.) were used.

Learning materials explaining scanning techniques for obtaining appropriate images and understanding normal and abnormal images for each US application were created for each learning objective shown in Table 1, and were provided to trainees one week before the start of the course. In the lectures, the instructors explained the learning objectives for each part and demonstrated normal and abnormal images using scanning video clips. In the hands-on sessions, the trainees had opportunities to scan live patients and/or simulators to practice their scanning skills under the instructors' supervision. During the hands-on training, the instructors 
Table 2 Teaching domains selected for inclusion in the pediatric POCUS training course

$\overline{\text { Pediatric POCUS training course (participant }}$ Educational content number)

August $2019(n=18)$

January $2020(n=13)$

used standardized materials to teach scanning techniques in each session in accordance with each learning objective in Table 1, and used video clips (cardiac, airway/lung, abdominal, ocular, and musculoskeletal US, and US-guided procedures) and scanned images of pediatric anatomical simulators (abdominal US) or chicken legs (musculoskeletal US) to illustrate abnormal images in each session. The course director monitored all the stations to ensure a standard quality of instruction.

\section{Trainees}

Physicians interested in using pediatric POCUS were recruited using marketing materials at the Japan Society of Point-of-Care Ultrasound meetings to be trainees in the halfday pediatric POCUS training course. Pre-registration was required for participation; however, prospective trainees who failed to register were allowed to register on-site.

\section{Questionnaire}

The questionnaire used in our study was created by referencing previously published studies on implementing a POCUS training course $[5,17,18]$ after discussion among the instructors (Table 3). Due to the difficulty of simultaneously evaluating all components of the Kirkpatrick model for each course, the overall satisfaction level with our training course and the trainees' self-efficacy level in each US application before and after the course were selected for assessment in this pilot study.

\section{Data collection}

Data were collected using printed materials, and pre- and post-training evaluation forms were completed by the trainees. All assessment forms, including items on the participants' background, such as their post-graduate year (PGY), experience participating in pediatric POCUS courses, experience using pediatric POCUS in daily practice, self-efficacy in pediatric POCUS use before and after the course, and overall satisfaction with the course were provided in Japanese (Table 3 ). The instructors were blinded to the written questionnaire.

\section{Measures}

The self-efficacy and satisfaction rates were assessed using a Likert scale from 0 (no confidence) to 100 (complete confidence) with 11-point intervals measuring confidence [19], and a Likert scale from 0 (no satisfaction) to 10 (full satisfaction), respectively, after the training.

\section{Statistical analysis}

The effect size of the difference in confidence level for pediatric POCUS was calculated using previous data on confidence levels obtained from a hands-on session at the 29th annual meeting of the Japanese Society of Emergency Pediatrics in 2015 [unpublished work]. With an effect size of the difference in self-assessed competency of 1.2 on a 5-point Likert scale, a sample size of 24 participants with a $5 \%$ significance level was calculated to obtain $90 \%$ power. The statistical analysis was performed using SPSS statistical software, version 18.0 (SPSS Inc. Chicago, IL, USA). Summary statistics were expressed as proportions with 95\% confidence intervals (CI), and tests for association were done using the paired $t$ test. $p \leq 0.05$ was considered to indicate statistical significance.

\section{Ethical considerations}

The present study was conducted in accordance with the Declaration of Helsinki (2013) and was approved by the Institutional Review Board of our hospital (Approval number 2019-b74). The aim of this study was inscribed on the questionnaires given to the trainees and was explained orally by the course director before the start of training. Participation was voluntary, and submission of the questionnaires was understood as the participants' consent to be enrolled. 
Table 3 Pre- and post-course survey

Pre-course survey (2019 August)

Questions

1. What is your training level?

a. Intern (PGY 1-2)

b. Fellow (PGY 3-5)

c. Attending physician (PGY $5<$ )

2. What is your specialty?

a. Pediatrics

b. Internal medicine

c. Emergency medicine

d. Anesthesiology

e. Intensive care medicine

f. Radiology

g. Other

3. Have you ever been trained in pediatric POCUS?

a. Yes b. No

4. Do you use ultrasonography in your daily practice?

a. Yes b. No

5. Are you confident of your skills in pediatric cardiac POCUS?

6. Are you confident of your skills in pediatric airway/lung POCUS?

7. Are you confident of your skills in POCUS for pediatric fractures?

8. Are you confident of your skills in pediatric soft tissue POCUS?

9. Are you confident of your skills in POCUS for pediatric shock?

10. Are you confident of your skills in pediatric ultrasound-guided procedures?

11. Are you confident of your overall skills in pediatric POCUS?

Post-course survey

Questions

1. What was beneficial for you in this course?

2. Do you have any suggestions for improving this course?

3. Are there any topics that you would like to learn about in the future?

4. After attending this course, have you become more confident of your skills in pediatric cardiac POCUS?

5. After attending this course, have you become more confident of your skills in pediatric airway/lung POCUS?

6. After attending this course, have you become more confident of your skills in POCUS for pediatric fractures?

7. After attending this course, have you become more confident of your skills in pediatric soft tissue POCUS?

8. After attending this course, have you become more confident of your skills in POCUS for pediatric shock?

9. After attending this course, have you become more confident of your skills in pediatric ultrasound-guided procedures?

10. After attending this course, have you become more confident of your overall skills in pediatric POCUS?

11. Overall, are you satisfied with this course?

\section{Results}

Of the 31 physicians enrolled, 25 completed all the surveys. Table 4 shows their demographic characteristics. Ten participants were in PGY 1-2, 13 were in PGY 3-5, and eight were in PGY 6 or higher. Pediatricians comprised $38.7 \%$ of the trainees (12/31), followed by interns at $22.6 \%(7 / 31)$ and internal medicine physicians at $16.1 \%$ (5/31). Fifteen participants $(48.3 \%)$ had previous experience of attending an educational course on POCUS, and $87.1 \%$ of the trainees responded that they used US in their daily practice. Table 5 shows that the overall self-efficacy level in pediatric POCUS use after training was significantly higher than in the precourse assessment at 86.0 (standard deviation [SD] 19.2) and 35.6 (SD 17.6), respectively (mean difference [95\% confidence interval] 49.6 [39.6-61.2]) $(p<0.05)$. For each component—including cardiac, abdominal, airway/lung, musculoskeletal, and ocular US, US-guided procedures, and shock management-the self-efficacy levels on the posttraining evaluation were significantly higher. The increase in 
Table 4 Trainee demographics

\begin{tabular}{lc}
\hline & $\%(n)$ \\
\hline PGY & \\
$1-2$ & $32.3(10 / 31)$ \\
$3-5$ & $41.9(13 / 31)$ \\
$5<$ & $25.8(8 / 31)$ \\
Subspecialty & \\
Pediatrics & $38.7(12 / 31)$ \\
Internal medicine & $16.1(5 / 31)$ \\
Family medicine & $6.5(2 / 31)$ \\
Anesthesiology & $6.5(2 / 31)$ \\
Cardiology & $3.2(1 / 31)$ \\
Rehabilitation & $3.2(1 / 31)$ \\
Emergency medicine & $3.2(1 / 31)$ \\
Internship & $22.6(7 / 31)$ \\
Previous training in POCUS & $48.4(15 / 31)$ \\
POCUS use in daily practice & $87.1(27 / 31)$ \\
\hline
\end{tabular}

$P G Y$ post-graduate year the self-efficacy level from before to after the course tended to be higher for musculoskeletal and ocular US than for cardiac and abdominal US, and the overall satisfaction rate on the post-training evaluation was high (Table 5). There was no difference in the self-efficacy level in terms of specialty (Table 6).

\section{Discussion}

To the best of our knowledge, the present study is the first to assess a comprehensive training course in pediatric POCUS in Japan. A pilot training course in pediatric POCUS was created on the basis of previously published guidelines and consensus statements [3, 4, 15], which include over 20 procedures that PEM physicians are required to master to treat any of a variety of physical conditions encountered in the course of their work [20]. In a study conducted in a pediatric emergency department (ED), a 1-day training course in POCUS use for pediatric soft tissue infections demonstrated improvement in the users' knowledge and skills
Table 5 Self-assessment of competency in pediatric POCUS

\begin{tabular}{lllll}
\hline $\begin{array}{l}\text { Educational domains, (respondent/ } \\
\text { participant number) }\end{array}$ & \multicolumn{2}{l}{$\begin{array}{l}\text { Self-efficacy score (mean } \\
\text { (SD)) }\end{array}$} & Mean difference (95\% CI) & $p$ value \\
\cline { 2 - 3 } & Pre & Post & \\
\hline Overall (25/31) & $35.6(17.6)$ & $86.0(19.2)$ & $49.6(39.6-61.2)$ & $<0.05$ \\
Cardiac (25/31) & $35.6(23.8)$ & $73.6(13.5)$ & $38.0(27.1-48.9)$ & $<0.05$ \\
Abdomen (9/13) & $27.7(18.6)$ & $63.3(26.9)$ & $35.6(21.1-50.0)$ & $<0.05$ \\
Airway/Lung (17/18) & $31.8(23.0)$ & $80.0(13.7)$ & $48.2(34.9-61.6)$ & $<0.05$ \\
Fracture (17/18) & $18.5(12.3)$ & $73.5(19.9)$ & $54.7(40.9-68.6)$ & $<0.05$ \\
Soft tissue (17/18) & $28.2(23.5)$ & $78.8(14.5)$ & $50.6(37.4-63.4)$ & $<0.05$ \\
Ocular (9/13) & $18.9(23.2)$ & $81.1(14.5)$ & $62.2(40.0-86.5)$ & $<0.05$ \\
Ultrasound-guided procedures (26/31) & $23.1(19.8)$ & $73.5(20.8)$ & $50.4(39.6-61.2)$ & $<0.05$ \\
Shock management (25/31) & $28.8(21.5)$ & $77.6(14.5)$ & $48.8(39.9-57.7)$ & $<0.05$ \\
& Satisfaction rate (mean & & \\
Overall (25/31) & (SD)) & & \\
\hline
\end{tabular}

POCUS point-of-care ultrasound, SD standard deviation, NA not applicable, 95\% CI 95\% confidence interval

Table 6 Self-efficacy in pediatric POCUS by specialty

\begin{tabular}{lll}
\hline & Pre-course confidence level (mean (SD)) & $p$ value \\
\hline Pediatrician & $38.9(13.7)$ & \\
Non-pediatrician & $37.5(19.5)$ & 0.56 \\
\hline & Post-course confidence level (mean (SD)) \\
\hline Pediatrician & $77.8(23.5)$ \\
Non-pediatrician & $90.6(13.4)$ \\
\hline
\end{tabular}

POCUS point-of-care ultrasound, SD standard deviation 
when examining cases of cellulitis and subcutaneous abscess [11]. Another study demonstrated that a web-based training course in US-guided nerve block in children increased physicians' comfort and intention levels with this procedure [6]. In addition, a 1- to 3-day training course on lung and cardiac US for pediatric intensivists and pediatric cardiologists showed a significant improvement in physicians' knowledge and skills in performing US and interpreting the findings [7-10, 12]. However, these studies focused on a narrow variety of applications. In contrast, a study conducted in a pediatric ED in Puerto Rico demonstrated that a 2-day course in pediatric POCUS including a broad spectrum of applications, such as cardiac, pulmonary, and soft tissue US, improved pediatricians' knowledge and confidence level in pediatric POCUS; the sample size of this study, however, was small [13]. Similarly, the present study demonstrated an improvement in the self-efficacy resulting from comprehensive training in pediatric POCUS use. Thus, our findings have the potential to promote the use of POCUS in the pediatric emergency setting in Japan.

The present study also found a significant increase in the trainees' self-efficacy levels in performing the procedure after training (Table 5). In our study, the self-efficacy level for ocular, fracture, and soft tissue US increased considerably between before and after the course, which was consistent with the findings of previously published studies of US training courses demonstrating that the self-efficacy level was lower in US applications that trainees had not experienced than in those they had, and that the self-efficacy level increased significantly after the course [5, 21, 22]. In addition, the hands-on session contributed greatly to enhancing trainees' efficacy level, as seen previously in a study of pediatric POCUS training [5]. In our survey, many participants expressed satisfaction with the hands-on session. An increase in the trainees' self-efficacy level in POCUS led to an increase in the frequency of their taking the initiative to perform US examinations in their practice, which will encourage further learning and skill improvements $[13,21]$. Therefore, the results of the current study demonstrated the effectiveness of our training course in facilitating pediatric POCUS use.

From the medical education perspective, the Kirkpatrick model was used to evaluate educational efficacy [23]. This model consists of levels 1 to 4 , each at which the trainees' respond to their learning experience: learning outcomes, such as increased knowledge, skills, and changes in attitude towards their training; changes in trainees' behavior and improvements in the quality of patient care; and the results and impacts of training are assessed [24]. The question in Table 3 asking "Overall, are you satisfied with this course?" aimed to assess the participants' satisfaction level (level 1 criteria), whereas questions such as "Are you confident of your skills in airway/lung ultrasound?" aimed to assess their self-efficacy level for each type of pediatric POCUS application (level 2 criteria). The considerable improvement in selfefficacy scores seen in all categories demonstrated that the training satisfied the level 2 (Learning) criteria. Although investigating the applicability of the POCUS techniques taught in the course to the participants' daily practice (level 3 ) and determining how these techniques might improve the quality of pediatric emergency care (level 4) are necessary to evaluate the efficacy of the course in an actual clinical setting, significant yet still preliminary progress was made by including a comprehensive range of procedures. The optimal duration of training is still unknown, but courses lasting one to three days are common [25]. Therefore, future training courses incorporating assessments at levels 3 and 4 of the Kirkpatrick model may be held for one to two days. Furthermore, during the current COVID-19 pandemic, most lectures and conferences have moved online or have combined faceto-face with online tutorials [26]. A previous study showed that there was no difference between web-based learning and face-to-face education in the acquisition of knowledge in EFAST use [27]. In addition, other studies demonstrated that online learning showed non-inferiority in terms of skill acquisition in cardiac and musculoskeletal US [28, 29]. Thus, online education can be a viable alternative to traditional face-to-face learning in pediatric POCUS training.

The present study had several limitations. First, the trainees' image acquisition and interpretation and skill retention in pediatric POCUS were not assessed due to the constraints on time and space imposed by the fact that the course was an adjunct to an academic conference. The emphasis thus fell on investigating the feasibility of a pilot study enrolling physicians with a variety of different backgrounds. A study investigating these components is currently being developed for the next training course. Second, the sample size was quite small. However, it was sufficient to demonstrate a significant improvement in the trainees' self-efficacy in performing POCUS, as seen in the sample size calculation based on our previous data. Third, physicians who participate in professional development activities such as skills training tend to show low self-efficacy in the pre-test setting; thus, selection bias may have entered into the present study. Fourth, in the hands-on sessions, young adult volunteers were used for scanning, which may have lessened the impact on educational objectives in pediatric POCUS. However, because recruiting pediatric volunteers was not feasible, pediatric anatomical simulators were used instead. Fifth, the improvement in self-efficacy levels may have been influenced by the Hawthorne effect. During the course, all the trainees were supervised by the instructors and the course director, possibly resulting in an overestimation of their self-assessment on the post-training survey. Therefore, as the next step, implementing an instructor training course to maintain the quality of teaching skills at a high level 
and planning a longer course in pediatric POCUS use that includes objective measures, such as an objective structured clinical examination (OSCE), computer-based tests, and a control group, may be desirable to evaluate trainees' skills more accurately [30].

\section{Conclusion}

The present study implemented a training course in pediatric POCUS and demonstrated a significant improvement in the trainees' self-efficacy level. The study revealed that comprehensive training has the potential to encourage physicians to use pediatric POCUS in their daily practice.

Acknowledgements We all thank Mr. James R. Valera for his assistance with editing this manuscript.

Author contributions TM conceptualized and designed the study, collected data, carried out the initial analysis, and drafted the initial manuscript. HT, ON, SF, and KI coordinated and supervised data collection and critically reviewed the manuscript for important intellectual content. All the authors approved the final manuscript as submitted and agree to be accountable for all aspects of the work.

\section{Declarations}

Conflict of interest Takaaki Mori, Osamu Nomura, Hirokazu Takei, Shinichi Fukuhara, and Ko Ichihashi have no financial relationships relevant of this article to disclose.

Ethical statements The aim of this study was inscribed on the questionnaires given to the trainees and was explained orally by the course director before the start of training. Participation was voluntary, and submission of the questionnaires was understood as the participants' consent to be enrolled.

\section{References}

1. Marin JR, Lewiss RE. Point-of-care ultrasonography by pediatric emergency medicine physicians. Pediatr Emerg Care. 2015;31:525.

2. Pearce MS, Salotti JA, Little MP, et al. Radiation exposure from CT scans in childhood and subsequent risk of leukaemia and brain tumours: a retrospective cohort study. Lancet. 2012;380:499-505.

3. American Academy Of Pediatrics CoPEM, Society For Academic Emergency Medicine AoEU, American College Of Emergency Physicians PEMC, World Interactive Network Focused On Critical U, Medicine AAOPCoPE. Point-of-care ultrasonography by pediatric emergency medicine physicians. Pediatrics. 2015;135:e1097-104.

4. Marin JR, Abo AM, Arroyo AC, et al. Pediatric emergency medicine point-of-care ultrasound: summary of the evidence. Crit Ultrasound J. 2016;8:16.

5. Alzayedi AS, Azizalrahman AA, AlMadi HA, et al. Use and education of point-of-care ultrasound in pediatric emergency medicine in Saudi Arabia. J Ultrasound Med. 2017;36:2219-25.

6. Bretholz A, Doan Q, Cheng A, et al. A presurvey and postsurvey of a web- and simulation-based course of ultrasound-guided nerve blocks for pediatric emergency medicine. Pediatr Emerg Care. 2012;28:506-9.

7. Ceresnak SR, Axelrod DM, Sacks LD, et al. Advances in pediatric cardiology boot camp: boot camp training promotes fellowship readiness and enables retention of knowledge. Pediatr Cardiol. 2017;38:631-40.

8. Conlon TW, Himebauch AS, Fitzgerald JC, et al. Implementation of a pediatric critical care focused bedside ultrasound training program in a large academic PICU. Pediatr Crit Care Med. 2015;16:219-26.

9. Gaspar HA, Morhy SS, Lianza AC, et al. Focused cardiac ultrasound: a training course for pediatric intensivists and emergency physicians. BMC Med Educ. 2014;14:25.

10. Good R, Orsborn J, Stidham T. Point-of-care ultrasound education for pediatric residents in the pediatric intensive care unit. MedEdPORTAL. 2018;14:10683.

11. Marin JR, Alpern ER, Panebianco NL, et al. Assessment of a training curriculum for emergency ultrasound for pediatric soft tissue infections. Acad Emerg Med. 2011;18:174-82.

12. Maskatia SA, Altman CA, Morris SA, et al. The echocardiography "boot camp": a novel approach in pediatric cardiovascular imaging education. J Am Soc Echocardiogr. 2013;26:1187-92.

13. Sepulveda-Ortiz V, Warkentine F, Starr-Seal R, et al. The effectiveness of a longitudinal ultrasound curriculum for general pediatricians working in a Puerto Rican emergency department: a pilot study. Ultrasound J. 2020;12:20.

14. Nishiya K, Sekiguchi S, Yoshimura H, et al. Good clinical teachers in pediatrics: the perspective of pediatricians in Japan. Pediatr Int. 2020;62:549-55.

15. Vieira RL, Hsu D, Nagler J, et al. Pediatric emergency medicine fellow training in ultrasound: consensus educational guidelines. Acad Emerg Med. 2013;20:300-6.

16. Pediatric Emergency and Critical Ultrasound Course (PECUS), https://www.winfocus.org/course/pediatric-emergency-criticalultrasound-course-pecus/.

17. Nomura O, Wiseman J, Sunohara M, et al. Japanese medical learners' achievement emotions: accounting for culture in translating Western medical educational theories and instruments into an asian context. Adv Health Sci Educ Theory Pract. 2021;26:1255-76.

18. Yamada T, Minami T, Soni NJ, et al. Skills acquisition for novice learners after a point-of-care ultrasound course: does clinical rank matter? BMC Med Educ. 2018;18:202.

19. Bandura A. Guide for constructing self-efficacy scales. In: Selfefficacy beliefs of adolescents 5. 2006;307-37.

20. Gorelick MH, Alpern ER, Alessandrini EA. A system for grouping presenting complaints: the pediatric emergency reason for visit clusters. Acad Emerg Med. 2005;12:723-31.

21. Allan CK, Tannous P, DeWitt E, et al. A pediatric cardiology fellowship boot camp improves trainee confidence. Cardiol Young. 2016;26:1514-21.

22. Wu WT, Chang KV, Han DS, et al. Musculoskeletal ultrasound workshops in postgraduate physician training: a pre- and post-workshop survey of 156 participants. BMC Med Educ. 2019;19:362.

23. Kirkpatrick D, Kirkpatrick J. Evaluating training programs: the four levels. Berrett-Koehler Publishers; 2006.

24. Heydari MR, Taghva F, Amini M, et al. Using Kirkpatrick's model to measure the effect of a new teaching and learning methods workshop for health care staff. BMC Res Notes. 2019;12:388.

25. Shefrin AE, Warkentine F, Constantine E, et al. Consensus core point-of-care ultrasound applications for pediatric emergency medicine training. AEM Educ Train. 2019;3:251-8.

26. Nomura O, Irie J, Park Y, et al. Evaluating effectiveness of youtube videos for teaching medical students CPR: solution to 
optimizing clinician educator workload during the COVID-19 pandemic. Int J Environ Res Public Health. 2021;18:7113.

27. Platz E, Goldflam K, Mennicke M, et al. Comparison of Webversus classroom-based basic ultrasonographic and EFAST training in 2 European hospitals. Ann Emerg Med. 2010;56:660-7.

28. Canty D, Barth J, Yang Y, et al. Comparison of learning outcomes for teaching focused cardiac ultrasound to physicians: a supervised human model course versus an eLearning guided self- directed simulator course. J Crit Care. 2019;49:38-44.

29. Das D, Kapoor M, Brown C, et al. Comparison of hands-on versus online learning in teaching ultrasound skills for Achilles tendon rupture: a pilot study. Cureus. 2020;12:e9021.
30. Nomura O, Onishi H, Park YS, et al. Predictors of performance on the pediatric board certification examination. BMC Med Educ. 2021;21:122.

Publisher's Note Springer Nature remains neutral with regard to jurisdictional claims in published maps and institutional affiliations. 